\title{
Search for the decay mode $B^{0} \rightarrow p p \bar{p} \bar{p}$
}

J. P. Lees, ${ }^{1}$ V. Poireau, ${ }^{1}$ V. Tisserand, ${ }^{1}$ E. Grauges,${ }^{2}$ A. Palano, ${ }^{3}$ G. Eigen, ${ }^{4}$ D. N. Brown, ${ }^{5}$ Yu. G. Kolomensky, ${ }^{5}$ M. Fritsch, ${ }^{6}$ H. Koch, ${ }^{6}$ T. Schroeder, ${ }^{6}$ C. Hearty, ${ }^{7,7 b}$ T. S. Mattison, ${ }^{7 b}$ J. A. McKenna, ${ }^{7 b}$ R. Y. So, ${ }^{7 b}$ V. E. Blinov, ${ }^{8 a, 8 b, 8 c}$ A. R. Buzykaev, ${ }^{8 a}$ V. P. Druzhinin, ${ }^{8 a, 8 b}$ V. B. Golubev, ${ }^{8 a, 8 b}$ E. A. Kozyrev ${ }^{8 a, 8 b}$ E. A. Kravchenko, ${ }^{8 a, 8 b}$ A. P. Onuchin, ${ }^{8 a, 8 b, 8 c}$

S. I. Serednyakov ${ }^{8 a, 8 b}$ Yu. I. Skovpen, ${ }^{8 a, 8 b}$ E. P. Solodov, ${ }^{8 a, 8 b}$ K. Yu. Todyshev, ${ }^{8 a, 8 b}$ A. J. Lankford, ${ }^{9}$ J. W. Gary, ${ }^{10}$ O. Long, ${ }^{10}$ A. M. Eisner, ${ }^{11}$ W. S. Lockman, ${ }^{11}$ W. Panduro Vazquez, ${ }^{11}$ D. S. Chao, ${ }^{12}$ C. H. Cheng, ${ }^{12}$ B. Echenard ${ }^{12}$ K. T. Flood, ${ }^{12}$ D. G. Hitlin, ${ }^{12}$ J. Kim, ${ }^{12}$ Y. Li, ${ }^{12}$ T. S. Miyashita, ${ }^{12}$ P. Ongmongkolkul, ${ }^{12}$ F. C. Porter, ${ }^{12}$ M. Röhrken, ${ }^{12}$ Z. Huard, ${ }^{13}$

B. T. Meadows, ${ }^{13}$ B. G. Pushpawela, ${ }^{13}$ M. D. Sokoloff, ${ }^{13}$ L. Sun, ${ }^{13, \dagger}$ J. G. Smith, ${ }^{14}$ S. R. Wagner, ${ }^{14}$ D. Bernard, ${ }^{15}$

M. Verderi, ${ }^{15}$ D. Bettoni, ${ }^{16 a}$ C. Bozzi, ${ }^{16 a}$ R. Calabrese, ${ }^{16 a, 16 b}$ G. Cibinetto ${ }_{16 a, 16 b}$ E. Fioravanti, ${ }^{16 a, 16 b}$ I. Garzia, ${ }^{16 a, 16 b}$ E. Luppi, ${ }^{16 a, 16 b}$ V. Santoro, ${ }^{16 a}$ A. Calcaterra, ${ }^{17}$ R. de Sangro, ${ }^{17}$ G. Finocchiaro, ${ }^{17}$ S. Martellotti,${ }^{17}$ P. Patteri, ${ }^{17}$ I. M. Peruzzi ${ }^{17}$ M. Piccolo, ${ }^{17}$ M. Rotondo, ${ }^{17}$ A. Zallo, ${ }^{17}$ S. Passaggio, ${ }^{18}$ C. Patrignani, ${ }^{18, *}$ H. M. Lacker, ${ }^{19}$ B. Bhuyan, ${ }^{20}{ }^{24}$ U. Mallik, ${ }^{21}$ C. Chen, ${ }^{22}$ J. Cochran, ${ }^{22}$ S. Prell, ${ }^{22}$ A. V. Gritsan, ${ }^{23}$ N. Arnaud, ${ }^{24}$ M. Davier, ${ }^{24}$ F. Le Diberder, ${ }^{24}$ A. M. Lutz, ${ }^{24}$ G. Wormser ${ }^{24}$ D. J. Lange, ${ }^{25}$ D. M. Wright, ${ }^{25}$ J. P. Coleman, ${ }^{26}$ E. Gabathuler, ${ }^{26, *}$ D. E. Hutchcroft, ${ }^{26}$ D. J. Payne, ${ }^{26}$ C. Touramanis, ${ }^{26}$ A. J. Bevan, ${ }^{27}$ F. Di Lodovico, ${ }^{27}$ R. Sacco, ${ }^{27}$ G. Cowan, ${ }^{28}$ Sw. Banerjee, ${ }^{29}$ D. N. Brown, ${ }^{29}$ C. L. Davis, ${ }^{29}$ A. G. Denig, ${ }^{30}$ W. Gradl, ${ }^{30}$ K. Griessinger, ${ }^{30}$ A. Hafner, ${ }^{30}$ K. R. Schubert, ${ }^{30}$ R. J. Barlow, ${ }^{31,}$ G. D. Lafferty, ${ }^{31}$ R. Cenci, ${ }^{32}$ A. Jawahery, ${ }^{32}$ D. A. Roberts, ${ }^{32}$ R. Cowan, ${ }^{33}$ S. H. Robertson, ${ }^{34 a, 34 b}$ R. M. Seddon, ${ }^{34 b}$ B. Dey ${ }^{35 a}$ N. Neri, ${ }^{35 a}$ F. Palombo, ${ }^{35 a, 35 b}$ R. Cheaib ${ }^{36}$ L. Cremaldi, ${ }^{36}$ R. Godang, ${ }^{36, \|}$ D. J. Summers, ${ }^{36}$ P. Taras, ${ }^{37}$ G. De Nardo, ${ }^{38}$ C. Sciacca ${ }^{38}$ G. Raven, ${ }^{39}$ C. P. Jessop, ${ }^{40}$ J. M. LoSecco ${ }^{40}$ K. Honscheid, ${ }^{41}$ R. Kass, ${ }^{41}$ A. Gaz, ${ }^{42 a}$ M. Margoni,${ }^{42 a, 42 b}$ M. Posocco, ${ }^{42 a}$ G. Simi, ${ }^{42 a, 42 b}$ F. Simonetto, ${ }^{42 a, 42 b}$ R. Stroili, ${ }^{42 a, 42 b}$ S. Akar, ${ }^{43}$ E. Ben-Haim, ${ }^{43}$ M. Bomben, ${ }^{43}$ G. R. Bonneaud, ${ }^{43}$ G. Calderini, ${ }^{43}$ J. Chauveau, ${ }^{43}$ G. Marchiori, ${ }^{43}$ J. Ocariz, ${ }^{43}$ M. Biasini, ${ }^{44,44 b}$ E. Manoni, ${ }^{44 a}$ A. Rossi, ${ }^{44 a}$ G. Batignani, ${ }^{45 a, 45 b}$ S. Bettarini, ${ }^{45,45 b}$ M. Carpinelli, ${ }^{45 a, 45 b,{ }^{4}}$ G. Casarosa, ${ }^{45 a, 45 b}$ M. Chrzaszcz, ${ }^{45 a}$ F. Forti, ${ }^{45 a, 45 b}$ M. A. Giorgi, ${ }^{45 a, 45 b}$ A. Lusiani, ${ }^{45 a, 45 c}$ B. Oberhof, ${ }^{45 a, 45 b}$ E. Paoloni, ${ }^{45 a, 45 b}$ M. Rama, ${ }^{45 a}$ G. Rizzo, ${ }^{45 a, 45 b}$ J. J. Walsh, ${ }^{45 a}$ L. Zani ${ }^{45 a, 45 b}$ A. J. S. Smith, ${ }^{46}$ F. Anulli, ${ }^{47 a}$ R. Faccini, ${ }^{47 a, 47 b}$ F. Ferrarotto, ${ }^{47 a}$ F. Ferroni, ${ }^{47 a, 47 b}$ A. Pilloni, ${ }^{47 a, 47 b}$ G. Piredda,${ }^{47 a,}{ }^{2}$ C. Bünger, ${ }^{48}$ S. Dittrich, ${ }^{48}$ O. Grünberg, ${ }^{48}$ M. Heß, ${ }^{48}$ T. Leddigg ${ }^{48}$ C. Voß, ${ }^{48}$ R. Waldi, ${ }^{48}$ T. Adye, ${ }^{49}$ F. F. Wilson, ${ }^{49}$ S. Emery, ${ }^{50}$ G. Vasseur ${ }^{50}$ D. Aston, ${ }^{51}$ C. Cartaro,${ }^{51}$ M. R. Convery, ${ }^{51}$ J. Dorfan, ${ }^{51}$ W. Dunwoodie, ${ }^{51}$ M. Ebert, ${ }^{51}$ R. C. Field ${ }^{51}$ B. G. Fulsom, ${ }^{51}$ M. T. Graham, ${ }^{51}$ C. Hast, ${ }^{51}$ W. R. Innes, ${ }^{51, *}$ P. Kim,${ }^{51}$

D. W. G. S. Leith, ${ }^{51}$ S. Luitz, ${ }^{51}$ D. B. MacFarlane, ${ }^{51}$ D. R. Muller, ${ }^{51}$ H. Neal,${ }^{51}$ B. N. Ratcliff, ${ }^{51}$ A. Roodman,${ }^{51}$

M. K. Sullivan, ${ }^{51}$ J. Va'vra, ${ }^{51}$ W. J. Wisniewski, ${ }^{51}$ M. V. Purohit, ${ }^{52}$ J. R. Wilson, ${ }^{52}$ A. Randle-Conde, ${ }^{53}$ S. J. Sekula, ${ }^{53}$ H. Ahmed, ${ }^{54}$ M. Bellis, ${ }^{55}$ P. R. Burchat,${ }^{55}$ E. M. T. Puccio,${ }^{55}$ M. S. Alam,${ }^{56}$ J. A. Ernst, ${ }^{56}$ R. Gorodeisky, ${ }^{57}$ N. Guttman,${ }^{57}$ D. R. Peimer, ${ }^{57}$ A. Soffer, ${ }^{57}$ S. M. Spanier, ${ }^{58}$ J. L. Ritchie, ${ }^{59}$ R. F. Schwitters, ${ }^{59}$ J. M. Izen,${ }^{60}$ X. C. Lou, ${ }^{60}$ F. Bianchi,${ }^{61 a, 61 b}$ F. De Mori, ${ }^{61 \mathrm{a}, 61 \mathrm{~b}}$ A. Filippi, ${ }^{61 \mathrm{a}}$ D. Gamba, ${ }^{61 \mathrm{a}, 61 \mathrm{~b}}$ L. Lanceri, ${ }^{62}$ L. Vitale ${ }^{62}$ F. Martinez-Vidal, ${ }^{63}$ A. Oyanguren, ${ }^{63}$ J. Albert, ${ }^{64 \mathrm{~b}}$ A. Beaulieu, ${ }^{64 \mathrm{~b}}$ F. U. Bernlochner, ${ }^{64 \mathrm{~b}}$ G. J. King, ${ }^{64 \mathrm{~b}}$ R. Kowalewski, ${ }^{64 \mathrm{~b}}$ T. Lueck, ${ }^{64 \mathrm{~b}}$ I. M. Nugent, ${ }^{64 \mathrm{~b}}$ J. M. Roney, ${ }^{64 \mathrm{~b}}$ R. J. Sobie, ${ }^{64 a, 64 b}$ N. Tasneem, ${ }^{64 b}$ T. J. Gershon, ${ }^{65}$ P. F. Harrison, ${ }^{65}$ T. E. Latham, ${ }^{65}$ R. Prepost, ${ }^{66}$ and S. L. Wu ${ }^{66}$

\section{(BABAR Collaboration)}

\author{
${ }^{1}$ Laboratoire d'Annecy-le-Vieux de Physique des Particules (LAPP), Université de Savoie, \\ CNRS/IN2P3, F-74941 Annecy-Le-Vieux, France \\ ${ }^{2}$ Universitat de Barcelona, Facultat de Fisica, Departament ECM, E-08028 Barcelona, Spain \\ ${ }^{3}$ INFN Sezione di Bari and Dipartimento di Fisica, Università di Bari, I-70126 Bari, Italy \\ ${ }^{4}$ University of Bergen, Institute of Physics, N-5007 Bergen, Norway \\ ${ }^{5}$ Lawrence Berkeley National Laboratory and University of California, Berkeley, California 94720, USA \\ ${ }^{6}$ Ruhr Universität Bochum, Institut für Experimentalphysik 1, D-44780 Bochum, Germany \\ ${ }^{7 a}$ Institute of Particle Physics, Vancouver, British Columbia, Canada V6T $1 Z 1$ \\ ${ }^{7 b}$ University of British Columbia, Vancouver, British Columbia, Canada V6T 1 Z1 \\ ${ }^{8 \mathrm{a}}$ Budker Institute of Nuclear Physics SB RAS, Novosibirsk 630090, Russia \\ ${ }^{8 \mathrm{~b}}$ Novosibirsk State University, Novosibirsk 630090, Russia \\ ${ }^{8 \mathrm{c}}$ Novosibirsk State Technical University, Novosibirsk 630092, Russia \\ ${ }^{9}$ University of California at Irvine, Irvine, California 92697, USA \\ ${ }^{10}$ University of California at Riverside, Riverside, California 92521, USA \\ ${ }^{11}$ University of California at Santa Cruz, Institute for Particle Physics, Santa Cruz, California 95064, USA \\ ${ }^{12}$ California Institute of Technology, Pasadena, California 91125, USA \\ ${ }^{13}$ University of Cincinnati, Cincinnati, Ohio 45221, USA \\ ${ }^{14}$ University of Colorado, Boulder, Colorado 80309, USA \\ ${ }^{15}$ Laboratoire Leprince-Ringuet, Ecole Polytechnique, CNRS/IN2P3, F-91128 Palaiseau, France
}




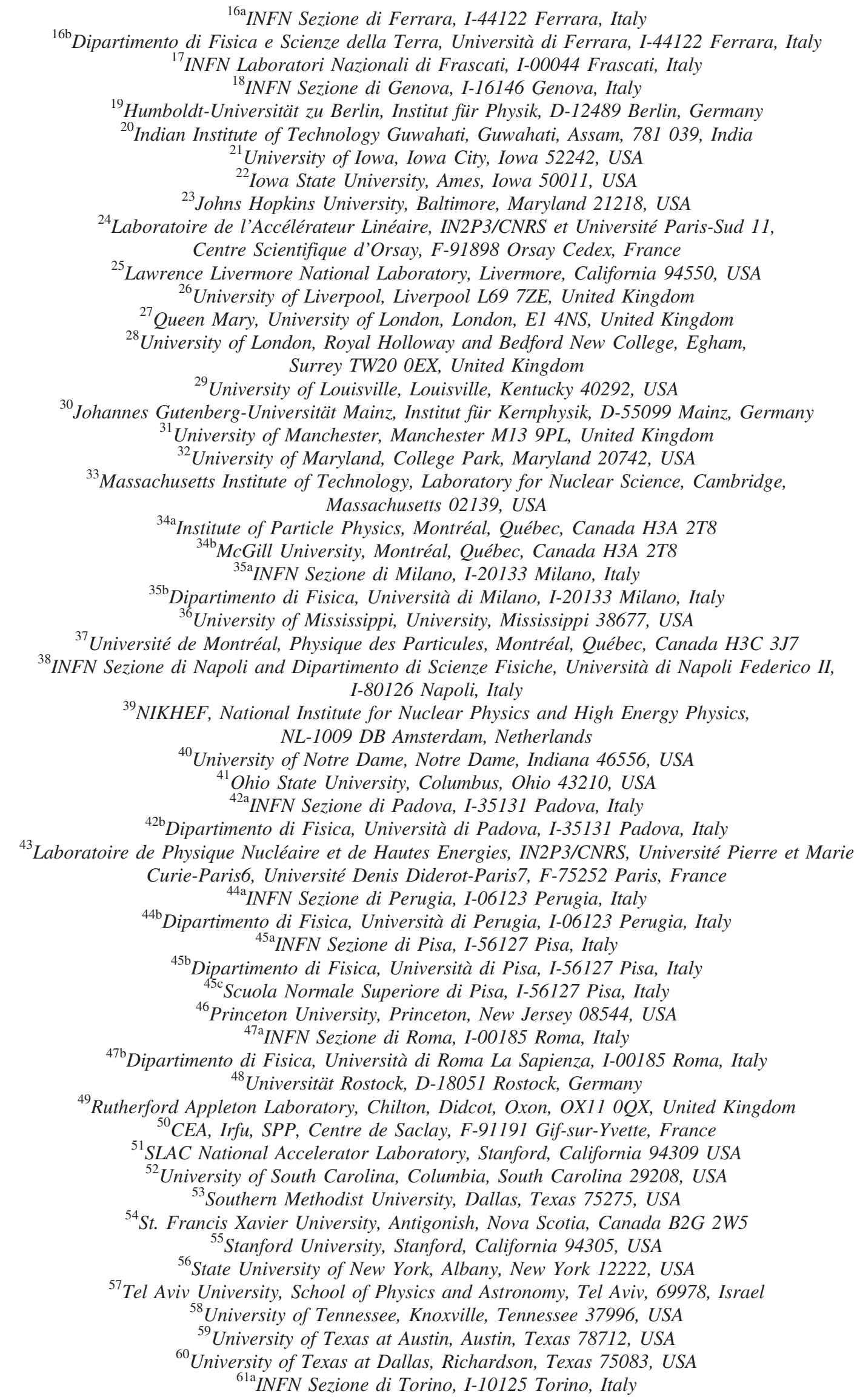




\author{
${ }^{61 \mathrm{~b}}$ Dipartimento di Fisica, Università di Torino, I-10125 Torino, Italy \\ ${ }^{62}$ INFN Sezione di Trieste and Dipartimento di Fisica, Università di Trieste, I-34127 Trieste, Italy \\ ${ }^{63}$ IFIC, Universitat de Valencia-CSIC, E-46071 Valencia, Spain \\ ${ }^{64 a}$ Institute of Particle Physics, Victoria, British Columbia, Canada V8W $3 P 6$ \\ ${ }^{64 \mathrm{~b}}$ University of Victoria, Victoria, British Columbia, Canada V8W $3 P 6$ \\ ${ }^{65}$ Department of Physics, University of Warwick, Coventry CV4 7AL, United Kingdom \\ ${ }^{66}$ University of Wisconsin, Madison, Wisconsin 53706, USA
}

(Received 29 March 2018; published 23 October 2018)

\begin{abstract}
A search is presented for the four-body decay $B^{0} \rightarrow p p \bar{p} \bar{p}$ in a sample of 471 million $B \bar{B}$ pairs collected with the BABAR detector, operated at the SLAC PEP-II asymmetric-energy $e^{+} e^{-}$collider. The center-ofmass energy is $10.58 \mathrm{GeV}$. From a fit to the distribution of the energy-substituted mass $m_{\mathrm{ES}}$, the branching fraction $\mathcal{B}\left(B^{0} \rightarrow p p \bar{p} \bar{p}\right)=(1.1 \pm 0.5 \pm 0.2) \times 10^{-7}$ is extracted, where the first uncertainty is statistical and the second is systematic. The significance of the signal, including the systematic uncertainty, is 2.9 standard deviations. The upper limit on the branching fraction is determined to be $2.0 \times 10^{-7}$ at $90 \%$ confidence level.
\end{abstract}

DOI: 10.1103/PhysRevD.98.071102

\section{INTRODUCTION}

The inclusive branching fraction of $B$ mesons decaying into final states with at least one baryon-antibaryon pair is approximately $7 \%$ [1], while the sum of all measurements of exclusive baryonic channels is less than $1 \%$ [2]. Recent measurements from the LHCb experiment [3-5] have raised new interest in this field. Studying exclusive baryonic decays of $B$ mesons provides a deeper insight into the mechanism of hadronization into baryons and may allow a better understanding of the threshold enhancement effect, which is a dynamical enhancement, relative to the pure phase space expectation, of the production rate of baryonantibaryon pairs at their invariant mass threshold. So far, this process is only qualitatively understood. Theoretical models, e.g., the quantum chromodynamics (QCD) sum rule [6] and the perturbative QCD approach [7], need validation and input from experimental data. Although the threshold effect is also observed in $B$ decays to charmed baryons [8], its effect is not as pronounced as in charmless three-body baryonic decays, where a peak at the threshold of the invariant baryon-antibaryon mass distribution was first observed $[9,10]$. This enhancement could explain the

\footnotetext{
*Deceased.

Now at Wuhan University, Wuhan 430072, China.

${ }^{*}$ Now at Università di Bologna and INFN Sezione di Bologna, I-47921 Rimini, Italy.

${ }^{\S}$ Now at University of Huddersfield, Huddersfield HD1 3DH, United Kingdom.

Now at University of South Alabama, Mobile, Alabama 36688, USA.

"Also at Università di Sassari, I-07100 Sassari, Italy.

Published by the American Physical Society under the terms of the Creative Commons Attribution 4.0 International license. Further distribution of this work must maintain attribution to the author(s) and the published article's title, journal citation, and DOI. Funded by SCOAP ${ }^{3}$.
}

hierarchy trend of the branching fractions for baryonic $B$ decays. It has been observed that, despite the phase space expectation, many three-body decays to one baryonantibaryon pair plus an accompanying meson have larger rates than their two-body counterpart, which is the decay to the same baryon-antibaryon pair but with no other particles in the final state, e.g., $\mathcal{B}\left(B^{-} \rightarrow \Lambda_{c} \bar{p} \pi^{-}\right)>\mathcal{B}\left(B^{0} \rightarrow \Lambda_{c} \bar{p}\right)$ and $\mathcal{B}\left(B^{-} \rightarrow p \bar{p} K^{-}\right)>\mathcal{B}\left(B^{0} \rightarrow p \bar{p}\right)$ [11]. Also, some three-body decays are suppressed compared to the fourbody case $[12,13]$. The phenomenological approaches describe these observations in terms of gluonic and fragmentation mechanisms [14] and pole models [15]. For final states with a $p \bar{p}$ pair, a threshold enhancement could possibly arise from an intermediate X(1835) baryonium resonance, as proposed in Ref. [16].

In this paper, we report on a search for $B^{0} \rightarrow p p \bar{p} \bar{p}$ decays (the inclusion of charge conjugate processes is implied throughout). The data were collected with the $B A B A R$ detector $[17,18]$ at the SLAC PEP-II asymmetricenergy $e^{+} e^{-}$collider. The decay of a $B$ meson to two baryon-antibaryon pairs has not yet been observed. No quantitative predictions for this specific process are yet available. The measurement of a four baryon decay mode would provide useful information to confront existing fragmentation models with the upper limit on branching fraction. Previously, we performed a search for $\bar{B}^{0} \rightarrow$ $\Lambda_{c}^{+} p \bar{p} \bar{p}$ decays, setting a $90 \%$ confidence level (C.L.) upper limit on the decay branching fraction of $2.8 \times 10^{-6}$ [19]. Based on this result, using a scaling factor to account for the Cabibbo suppression for the $b \rightarrow u$ decay, and also taking into account the larger phase space of the final state, we estimate the branching fraction for the $B^{0} \rightarrow p p \bar{p} \bar{p}$ decay mode to be on the order of $10^{-7}$ at the maximum. We use this assumption to optimize the selection criteria. The threshold effect has been found to be enhanced for large values of $q$, which is the available momentum in the rest 
frame of the decaying $B$, with no visible enhancement for $q$ values below about $200 \mathrm{MeV} / c$ [20]. This feature could explain the absence of an observed signal in $\bar{B}^{0} \rightarrow$ $\Lambda_{c}^{+} p \bar{p} \bar{p}$ decays and at the same time could enhance the branching fraction for $B^{0} \rightarrow p p \bar{p} \bar{p}$. Moreover, the $B^{0} \rightarrow$ $p p \bar{p} \bar{p}$ decay rate may benefit from the low-invariant-mass enhancement of the double $p \bar{p}$ system and from presence of nontrivial intermediate bound states [21].

\section{BABAR DETECTOR AND DATA SETS}

The analysis is based on the full data set collected with $B A B A R$ at center-of-mass energy $10.58 \mathrm{GeV}$, corresponding to the peak of the $\Upsilon(4 S)$ resonance. The event sample contains $N_{B \bar{B}}=471 \times 10^{6} B \bar{B}$ pairs, corresponding to integrated luminosity of $424 \mathrm{fb}^{-1}$ [22]. Charged-particle momenta are measured by means of a five-layer doublesided silicon vertex tracker and a 40-layer multiwire drift chamber, both operating in the $1.5 \mathrm{~T}$ magnetic field of a superconducting solenoid. The particle identification (PID) for protons, kaons, and pions uses the specific energy loss measured in the tracking devices and the measurement of the Cherenkov angle provided by the internally reflecting, ring-imaging Cherenkov detector. We use Monte Carlo (MC) simulated events of the processes $e^{+} e^{-} \rightarrow B \bar{B}$, where the $B$ mesons decay generically according to known branching fractions and decay amplitudes [2], and $e^{+} e^{-} \rightarrow$ $q \bar{q}$ (with $q=u, d, s, c$ ) to model the background. These samples correspond to at least three times the integrated luminosity of the data. In addition, we generate a sample of 687000 signal decays $e^{+} e^{-} \rightarrow B^{0} \bar{B}^{0}$, where one of the $B$ mesons decays into $p p \bar{p} \bar{p}$ (referred to as the signal MC sample). The kinematics of the decay products of the signal decay are simulated according to phase space. Monte Carlo events are simulated with the EvTGen and JetSET event generators [23,24], with the response of the detector simulated using the GEANT4 suite of programs [25]. Signal and background MC samples are used for the signal efficiency determination and for the modeling of the signal and background distributions.

\section{EVENT RECONSTRUCTION}

A $B$ meson candidate is reconstructed by combining four charged tracks, two identified as protons and two as antiprotons, kinematically fitting them to a common vertex and requiring the fit probability to exceed $0.1 \%$. The direction of the reconstructed $B$ meson is required to originate from the interaction region, which is constrained to the beam-spot size in the laboratory frame. Tracks are rejected if the combination of two oppositely charged tracks is found to be consistent with $K_{S}^{0}$ or $\Lambda$ hypotheses.

Loose preselection requirements are applied to the kinematic variables [20] $m_{\mathrm{ES}}=\sqrt{\left(E_{\text {beam }}^{*}\right)^{2}-\left(\vec{p}_{\mathrm{B}}^{*}\right)^{2}}>$ $5.2 \mathrm{GeV} / c^{2}$ and $\left|\Delta E=E_{\mathrm{B}}^{*}-E_{\text {beam }}^{*}\right|<0.2 \mathrm{GeV}$, where $\vec{p}_{\mathrm{B}}^{*}$ and $E_{\mathrm{B}}^{*}$ are, respectively, the momentum and energy of the reconstructed $B$ candidate in the $\mathrm{CM}$ frame, and $E_{\text {beam }}^{*}$ is half the CM energy. The study is performed as a blind analysis, which means that the selection is optimized without examining the data in the signal region, $5.27<m_{\mathrm{ES}}<5.29 \mathrm{GeV} / c^{2}$.

The PID efficiency for protons with mean momentum in the laboratory frame at the order of $1 \mathrm{GeV} / c$ is larger than 99\% and the misidentification of kaons and pions as protons is less than $1 \%$ [26]. The difference between the PID performance in data and simulation is evaluated using events from high-purity channels, which form the control samples (CS) for a given particle type. For example, events with $\Lambda \rightarrow p \pi^{-}$decays form the CS for the validation of proton PID, $K_{S}^{0} \rightarrow \pi^{+} \pi^{-}$for pion PID, and $D^{*+} \rightarrow$ $\pi^{+} D^{0}\left(D^{0} \rightarrow K^{-} \pi^{+}\right)$for kaon PID. The PID efficiency of the MC-simulated events is corrected to match that observed in data by applying the weight $\epsilon_{\mathrm{CS} \text {,Data }} / \epsilon_{\mathrm{CS}, \mathrm{MC}}$, where $\epsilon_{\mathrm{CS} \text {,Data }}$ and $\epsilon_{\mathrm{CS}, \mathrm{MC}}$ are the PID efficiencies evaluated from the CS in data and simulation, respectively.

\section{BACKGROUND REJECTION}

After applying the particle identification and preselection requirements, the fraction of selected signal candidates where at least one track has been misidentified is less than $0.2 \%$ in simulated signal events. The main background is combinatorial, from genuine protons in continuum $\left(e^{+} e^{-} \rightarrow q \bar{q}\right)$ events. The continuum background is further reduced by imposing a signal-like selection on the output of a multivariate boosted decision tree (BDT) algorithm. The BDT classifier uses the following input variables: $\Delta E$, $\cos \theta_{B}^{*}$, with $\theta_{B}^{*}$ the polar angle of the $B$ meson candidate with respect to the beam axis in the $\mathrm{CM}$ frame, and the event shape variables $R_{2}$ and $\left|\cos \theta_{\mathrm{TH}}\right|$, where $R_{2}$ is the ratio of the second to the zeroth Fox Wolfram moments [27] and $\theta_{\mathrm{TH}}$ is the angle between the thrust axis [28] of the $B$ candidate and that of the rest of the event in the $\Upsilon(4 S)$ rest frame. These kinematic and topological variables are effective in discriminating between spherically shaped events from $B \bar{B}$ decays and jet-like $q \bar{q}$ events.

In the BDT output, signal (background) events peak at positive (negative) values (Fig. 1). The optimal selection on the BDT output is determined by maximizing the figure of merit $S / \sqrt{S+B}$, where $S$ and $B$ are the number of expected signal and background events, respectively. The number of signal events is estimated assuming the signal branching fraction of $10^{-7}$ mentioned above. The selection is optimized using the MC samples and is validated by comparing the distributions for the background MC samples to the data in the control region $m_{\mathrm{ES}}<5.27 \mathrm{GeV} / c^{2}$. The total number of selected data events in the interval $5.2<m_{\mathrm{ES}}<5.3 \mathrm{GeV} / c^{2}$ is 117 . The signal efficiency, evaluated from simulation, is found to be $\epsilon=0.2068 \pm$ 0.0004 (stat).

It is important to notice that the only input from the simulation for this analysis is the signal efficiency, 


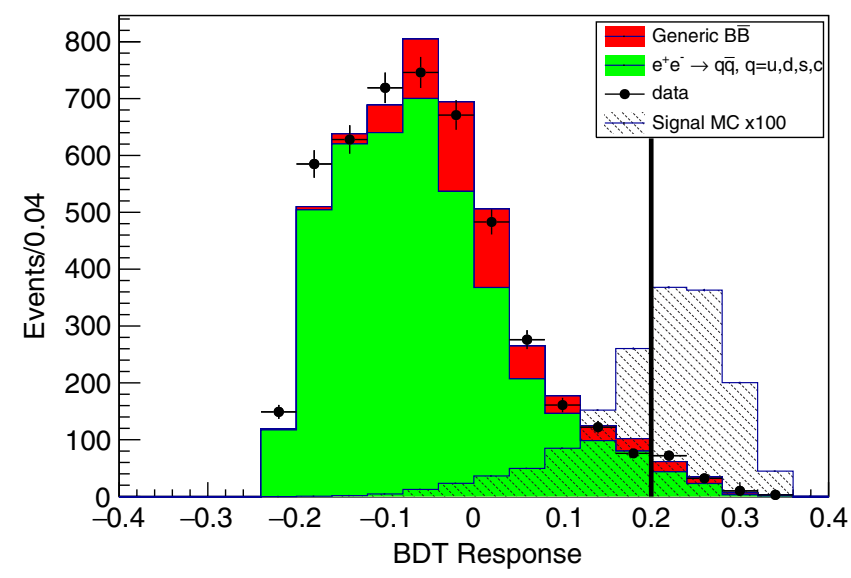

FIG. 1. The BDT output distribution for simulated signal (shaded histogram) and background (filled histogram) events. The two background components, from continuum and generic $B \bar{B}$ events, are stacked, with the total background prediction scaled to correspond to the number of selected events in the data. For purposes of visibility, the signal distribution has been multiplied by a factor of 100 . The selection on the BDT output is indicated by the black vertical line.

estimated on the simulated events of the processes $e^{+} e^{-} \rightarrow$ $B \bar{B}$ produced with EvTGEN, where one $B$ meson decays into the signal signature. The signal yield extraction does not rely on the MC description of the continuum background processes. In fact, the background shape in the final fit is extracted from the data, and therefore a potential discrepancy does not affect the measurement. Moreover, previous $B A B A R$ analyses show that the used input variables for the BDT algorithm developed in this work are well modeled for $B \bar{B}$ events. Finally, for any possible remaining discrepancies, dedicated systematic uncertainties are assigned, as explained later in this article. The uncertainty in the signal efficiency due to the BDT selection performed on these distributions is lower than 3\% and it demonstrates that the potential mismatch is under control.

We investigate the potential presence of peaking backgrounds from the baryonic modes $B \rightarrow p \bar{p} h^{+} h^{-}$, recently measured by the LHCb Collaboration [4], where $h$ is a charged kaon or pion. These decays can potentially enter the background if the $h^{+} h^{-}$pair is erroneously identified as a $p \bar{p}$ pair. This background is evaluated by applying the event selection to the simulated MC samples for the modes

TABLE I. Potential peaking background modes. Branching fractions [4], selection efficiencies, and the number of expected events at the data luminosity are reported.

\begin{tabular}{lccc}
\hline \hline Decay mode & $\mathcal{B}\left(10^{-6}\right)$ & $\begin{array}{c}\text { Selection efficiency } \\
\left(\epsilon_{p \bar{p} h^{+} h^{-}}\right)\end{array}$ & $N_{p \bar{p} h^{+} h^{-}}$ \\
\hline$p \bar{p} \pi \pi$ & $2.7 \pm 0.4$ & $(5 \pm 2) \times 10^{-6}$ & $6.3 \times 10^{-3}$ \\
$p \bar{p} K K$ & $0.11 \pm 0.03$ & $(1.5 \pm 0.7) \times 10^{-5}$ & $7.8 \times 10^{-4}$ \\
$p \bar{p} K \pi$ & $5.9 \pm 0.6$ & $(1.4 \pm 0.4) \times 10^{-5}$ & $3.9 \times 10^{-3}$ \\
\hline \hline
\end{tabular}

reported in Table I and determining the selection efficiencies $\epsilon_{p \bar{p} h^{+} h^{-}}$. The number of expected background events in the data for each channel is estimated as $N_{p \bar{p} h^{+} h^{-}}=$ $\epsilon_{p \bar{p} h^{+} h^{-}} \mathcal{B} N_{B \bar{B}}$ (Table I), where $\mathcal{B}$ is the branching fraction measured in Ref. [4] and $N_{B \bar{B}}$ is the total number of $B \bar{B}$ pairs in the initial data sample. The expected contamination from these decays is found to be negligible.

\section{SIGNAL YIELD EXTRACTION}

To describe the $m_{\mathrm{ES}}$ distribution in data, we use a probability density function (PDF) corresponding to the sum of the signal and the background components. The signal component is described by a Gaussian function, whose mean and width are fixed to values determined from a fit to simulated signal events. The combinatorial background component is described by the empirical ARGUS function [29], which depends on two parameters: a shape parameter and a cutoff parameter. The cutoff parameter is set equal to the endpoint in the $m_{\mathrm{ES}}$ spectrum, $5.289 \mathrm{GeV} / c^{2}$. The shape parameter is determined in the fit, along with the signal and background event yields, $N_{\text {sig }}$ and $N_{\text {bkg }}$, respectively.

The signal yield is extracted by performing an unbinned extended maximum likelihood fit to the $m_{\mathrm{ES}}$ distribution in the range $5.2<m_{\mathrm{ES}}<5.3 \mathrm{GeV} / c^{2}$ (Fig. 2). The logarithm of the extended likelihood is written as

$$
\begin{aligned}
\log L\left(N_{\mathrm{sig}}, N_{\mathrm{bkg}} ; x\right)= & -\left(N_{\mathrm{sig}}+N_{\mathrm{bkg}}\right)+\sum_{i=1}^{n} \log \left(N_{\mathrm{sig}} \cdot f_{\mathrm{sig}}\left(x_{i}\right)\right. \\
& \left.+N_{\mathrm{bkg}} \cdot f_{\mathrm{bkg}}\left(x_{i}\right)\right),
\end{aligned}
$$



FIG. 2. Fit to the data $m_{\mathrm{ES}}$ distribution (dots) in the interval $5.2<m_{\mathrm{ES}}<5.3 \mathrm{GeV} / c^{2}$. The bottom plot shows the pull distribution, which is the bin-by-bin difference between the data and fitted distribution normalized by the corresponding statistical uncertainty from the fit. 
TABLE II. Experimental inputs used for the branching fraction calculation.

\begin{tabular}{lcc}
\hline \hline Experimental input & Value & Statistical uncertainty \\
\hline$N_{\text {sig }}$ & 11.1 & 4.6 \\
$N_{B \bar{B}}$ & $470.88 \times 10^{6}$ & $0.12 \times 10^{6}$ \\
\hline \hline
\end{tabular}

where $x$ corresponds to the measured $m_{\mathrm{ES}}$ distribution and the $f_{j}(x)$ are the corresponding PDFs for the signal and background components. The sum of the signal and background yields is constrained to the total number of observed events $n$. The result is $N_{\text {sig }}=11.1 \pm 4.6$ (stat) events, from which the corresponding branching fraction is calculated as:

$\mathcal{B}\left(B^{0} \rightarrow p p \bar{p} \bar{p}\right)=\frac{N_{\text {sig }}}{\epsilon 2 N_{B^{0} \bar{B}^{0}}}=(1.14 \pm 0.47) \times 10^{-7}$,

where the uncertainty is statistical only and we assume equal production of $B^{0} \bar{B}^{0}$ and $B^{+} B^{-}$in $\Upsilon(4 S)$ decays. Therefore, $2 N_{B^{0} \bar{B}^{0}}=N_{B \bar{B}}$, where $N_{B \bar{B}}$ is the total number of $B \bar{B}$ pairs in the initial data sample. The value of 2 takes into account that the charge-conjugate decay is also reconstructed. The experimental values for $N_{\text {sig }}$ and $N_{B \bar{B}}$ are listed in Table II.

To evaluate the statistical significance of the branching fraction result, we fit the data under a background-only hypothesis and determine the corresponding change $\sqrt{-2(\Delta \ln L)}$ with respect to the standard fit, where $L$ is the likelihood function. The statistical significance is found to be 2.9 standard deviations. The systematic uncertainty in the ARGUS cutoff is taken into account and is found to not affect the signal significance.

\section{SYSTEMATIC UNCERTAINTIES}

Systematic uncertainties in the branching fraction arise from the fit procedure, from the uncertainty in $N_{B \bar{B}}$, and from the uncertainty in the signal efficiency. The relative systematic uncertainties for the considered sources are listed in Table III.

Potential systematic uncertainties associated with the fit procedure arise from the choice made for the signal PDF shape and from variation of the parameters held constant in the fit. Variations of the form chosen to model the signal PDF are found to have a negligible impact on the result, while the uncertainty associated with the ARGUS cutoff value, evaluated by varying the cutoff value within its uncertainty of $0.5 \mathrm{MeV} / c^{2}$, is $0.9 \%$

The systematic uncertainty in $N_{B \bar{B}}$ is estimated to be $0.6 \%$ [30].

To determine the systematic uncertainty in the signal efficiency, several sources are taken into account: the statistical uncertainty from the MC samples, the PID performance, the track finding efficiency, the BDT method,
TABLE III. Relative systematic uncertainties in the signal branching fraction. The total systematic uncertainty is determined by summing the individual contributions in quadrature.

\begin{tabular}{llc}
\hline \hline Variable & \multicolumn{1}{c}{ Source } & $\begin{array}{r}\text { Relative systematic } \\
\text { uncertainty }(\%)\end{array}$ \\
\hline$N_{B \bar{B}}$ & $B$ counting & 0.6 \\
$N_{\text {sig }}$ & ARGUS cutoff & 0.9 \\
$\epsilon$ & MC statistics & 0.2 \\
$\epsilon$ & PID efficiency & 0.9 \\
$\epsilon$ & Track finding efficiency & 0.9 \\
$\epsilon$ & BDT selection & 2.2 \\
$\epsilon$ & Decay model & 14 \\
Total & & 15 \\
\hline \hline
\end{tabular}

and the decay model used for the generation of the signal MC sample. The finite size of the signal MC sample results in a relative systematic uncertainty of $0.2 \%$. The PID performance contribution is taken as the effect of the full data-to-MC correction mentioned above and corresponds to a relative uncertainty of $0.9 \%$. The systematic uncertainty related to the track finding efficiency is a function of the particle momentum [31] and amounts to $0.9 \%$ for protons of approximately $1 \mathrm{GeV} / c$ momentum [31]. The systematic uncertainty in the signal efficiency introduced by the BDT method is evaluated by reweighting, separately for each of the four input variables of the BDT classifier, the shape of the MC distribution to match that observed in data. The weights are calculated in the control region $m_{\mathrm{ES}}<5.27 \mathrm{GeV} / c^{2}$, before the BDT selection, as the binby-bin ratio between data and $\mathrm{MC}$ events, and are applied to the corresponding distribution of the signal MC sample. The difference in the efficiency computed with and without the weights applied provides a systematic uncertainty of $2.2 \%$.

The systematic uncertainty related to the unknown dynamics of $B^{0} \rightarrow p p \bar{p} \bar{p}$ decays is evaluated by comparing the pure phase space decay MC sample to a model in which the decay proceeds through an intermediate spinless resonance, $B \rightarrow X(\rightarrow p \bar{p}) X(\rightarrow p \bar{p})$. Weights, binned in the four-dimensional space of the magnitudes of the momenta of the four tracks, are obtained by dividing the momentum distribution resulting from the resonant model by that from the phase space model, and are applied to the proton momentum distribution of the signal MC. The systematic uncertainty is obtained from the difference in the efficiency computed from the weighted and unweighted samples. The largest relative difference is obtained for an $X$ mass of $2.6 \mathrm{GeV} / c^{2}$, assuming a null width in order to compute the most extreme case. It amounts to $14 \%$ and is the largest contribution to the total systematic uncertainty.

\section{RESULTS}

The final result for the branching fraction is $\mathcal{B}\left(B^{0} \rightarrow p p \bar{p} \bar{p}\right)=\left(1.14 \pm 0.47_{\text {stat }} \pm 0.17_{\text {sys }}\right) \times 10^{-7}$. 
We use pseudoexperiments to establish the upper limit on the branching fraction taking into account the systematic variation. The signal yield $N_{\text {sig }}$ is varied according to the signal PDF computed from the fitted likelihood function and the signal efficiency $\epsilon$ and $N_{B \bar{B}}$ are randomly smeared according to two Gaussian distributions with mean 0.2068 and with width 0.03 , and with mean $470.88 \times 10^{6}$ and with width $2.83 \times 10^{6}$, respectively, corresponding to their absolute total uncertainties. For each pseudoexperiment, the branching fraction is calculated from the input values for $N_{\text {sig }}, N_{B \bar{B}}$, and $\epsilon$ randomly selected from the above defined PDFs and then its distribution is integrated up to $90 \%$ C.L., which yields an upper limit on the branching fraction of $2.0 \times 10^{-7}$.

In summary, we have performed a search for $B$ meson decays to the $p p \bar{p} \bar{p}$ final state, obtaining 11.1 signal events. The significance of the result, including both the statistical and systematic uncertainties, is 2.9 standard deviations. The branching fraction is measured to be $\mathcal{B}=(1.1 \pm 0.5$ (stat) \pm 0.2 (syst) $) \times 10^{-7}$. The corresponding $90 \%$ C.L. upper limit is $\mathcal{B}\left(B^{0} \rightarrow p p \bar{p} \bar{p}\right)<2.0 \times 10^{-7}$. Our result can provide important input for QCD models of hadronization and improve understanding of the threshold enhancement effect.

\section{ACKNOWLEDGMENTS}

We are grateful for the extraordinary contributions of our PEP-II colleagues in achieving the excellent luminosity and machine conditions that have made this work possible. The success of this project also relies critically on the expertise and dedication of the computing organizations that support $B A B A R$. The collaborating institutions wish to thank SLAC for its support and the kind hospitality extended to them. This work is supported by the U.S. Department of Energy and National Science Foundation, the Natural Sciences and Engineering Research Council (Canada), the Commissariat à l'Energie Atomique and Institut National de Physique Nucléaire et de Physique des Particules (France), the Bundesministerium für Bildung und Forschung and Deutsche Forschungsgemeinschaft (Germany), the Istituto Nazionale di Fisica Nucleare (Italy), the Foundation for Fundamental Research on Matter (The Netherlands), the Research Council of Norway, the Ministry of Education and Science of the Russian Federation, Ministerio de Economía y Competitividad (Spain), the Science and Technology Facilities Council (United Kingdom), and the Binational Science Foundation (U.S.-Israel). Individuals have received support from the Marie-Curie IEF program (European Union) and the A. P. Sloan Foundation (USA).
[1] H. Albrecht et al. (ARGUS Collaboration), Z. Phys. C 56, 1 (1992).

[2] C. Patrignani et al. (Particle Data Group Collaboration), Chin. Phys. C 40, 100001 (2016).

[3] R. Aaij et al. (LHCb Collaboration), Phys. Rev. Lett. 119, 232001 (2017).

[4] R. Aaij et al. (LHCb Collaboration), Phys. Rev. D 96, 051103 (2017).

[5] R. Aaij et al. (LHCb Collaboration), Phys. Rev. Lett. 119, 041802 (2017).

[6] V. L. Chernyak and I. R. Zhitnitsky, Nucl. Phys. 345, 137 (1990).

[7] X.-G. He, T. Li, X.-Q. Li, and Y.-M. Wang, Phys. Rev. D 75, 034011 (2007).

[8] N. Gabyshev et al. (Belle Collaboration), Phys. Rev. Lett. 97, 242001 (2006).

[9] M. Z. Wang et al. (Belle Collaboration), Phys. Rev. Lett. 92, 131801 (2004).

[10] K. Abe et al. (Belle Collaboration), Phys. Rev. Lett. 88, 181803 (2002).

[11] H. Y. Cheng and K. C. Yang, Phys. Rev. D 66, 014020 (2002).

[12] W. S. Hou and A. Soni, Phys. Rev. Lett. 86, 4247 (2001).

[13] P. del Amo Sanchez et al. (BABAR Collaboration), Phys. Rev. D 85, 092017 (2012).

[14] J. L. Rosner, Phys. Rev. D 68, 014004 (2003).

[15] M. Suzuki, J. Phys. G 34, 283 (2007).
[16] A. Datta and P. J. O'Donnell, Phys. Lett. B 567, 273 (2003).

[17] B. Aubert et al. (BABAR Collaboration), Nucl. Instrum. Methods Phys. Res., Sect. A 479, 1 (2002).

[18] B. Aubert et al. (BABAR Collaboration), Nucl. Instrum. Methods Phys. Res., Sect. A 729, 615 (2013).

[19] J. P. Lees et al. (BABAR Collaboration), Phys. Rev. D 89, 071102 (2014).

[20] A. J. Bevan et al. (BABAR and Belle Collaborations), Eur. Phys. J. C 74, 3026 (2014).

[21] J. P. Lees et al. (BABAR Collaboration), Phys. Rev. D 87, 092005 (2013).

[22] J. P. Lees et al. (BABAR Collaboration), Nucl. Instrum. Methods Phys. Res., Sect. A 726, 203 (2013).

[23] D. J. Lange, Nucl. Instrum. Methods Phys. Res., Sect. A 462, 152 (2001).

[24] T. Sjöstrand, Comput. Phys. Commun. 82, 74 (1994).

[25] S. Agostinelli et al. (GEANT4 Collaboration), Nucl. Instrum. Methods Phys. Res., Sect. A 506, 250 (2003).

[26] B. Aubert et al. (BABAR Collaboration), Nucl. Instrum. Methods Phys. Res., Sect. A 729, 615 (2013).

[27] G. C. Fox and S. Wolfram, Nucl. Phys. B149, 413 (1979); B157, 543(E) (1979).

[28] E. Farhi, Phys. Rev. Lett. 39, 1587 (1977).

[29] H. Albrecht et al., Phys. Lett. B 241, 278 (1990).

[30] G. D. McGregor, B Counting at BABAR,arXiv:0812.1954.

[31] T. Allmendinger et al., Nucl. Instrum. Methods Phys. Res., Sect. A 704, 44 (2013). 